\title{
The Dugdale solution for two unequal straight cracks weakening in an infinite plate
}

\author{
R R BHARGAVA ${ }^{1}$ and SHEHZAD HASAN ${ }^{2}$ \\ ${ }^{1}$ Department of Mathematics, Indian Institute of Technology Roorkee, \\ Roorkee 247667 \\ ${ }^{2}$ Department of Mathematics, Jamia Millia Islamia, New Delhi 110025 \\ e-mail: rajrbfma@iitr.ernet.in; drshehzadhasan@gmail.com
}

MS received 15 February 2008; revised 8 June 2009; accepted 7 October 2009

\begin{abstract}
A crack arrest model is proposed for an infinite elastic perfectlyplastic plate weakened by two unequal, quasi-static, collinear straight cracks. The Dugdale model solution is obtained for the above problem when the developed plastic zones are subjected to normal cohesive quadratically varying yield point stress. Employing complex variable technique and the superposition principle the desired solution is obtained. A qualitative study of load required to arrest the plastic zones opening with respect to affecting parameters viz. inter-crack distance, plastic zone and crack length is carried out. The results obtained are presented graphically.
\end{abstract}

Keywords. Crack arrest; Dugdale model; plastic zone.

\section{Introduction}

The study of collinear crack problems becomes imperative from the view point of its occurrence in many real life situations. Such situations are encountered in rocks science to material manufacturing and to mechanical engineering, etc. For example, rock media is practically always contains a network of cracks, defects in the form of crack or cavity or both nucleate in course of manufacturing process and for service life of mechanical components, etc. To be able to predict the integrity in such situation it is desired to model to such cases and investigate.

Dugdale (1960) proposed a simple model when a straight slit weakening a plate is opened in Mode-I type deformation under tensile force applied remotely. The rims of the plastic zones formed were taken to be closed by normal uniform constant yield point stress. This model was modified by Harrop (1978) when rims of the plastic zones were closed by cohesive normal yield point stress distribution varying as an arbitrary second order polynomial. Theocaris (1983) found Dugdale model solution for two collinear straight cracks. Bhargava \& Hasan (2001) have proposed a crack closure model for a circular arc cracks weakening a plate. The model was modified by Wang et al (2002) for the case when applied load was characterized by the specific remote strain. Beretta \& Carboni (2005) used enriched finite element penalty functions method for modelling interface crack with contact zones. An analytic solution 
for two equal length collinear strip yield cracks weakening an unbounded plate has been obtained by Collins \& Cartwright (2001) using complex variable technique developed by Muskhelishvili (1953). The problem was further extended by Nishimura (2002) for the case of two collinear unequal straight cracks weakening an infinite sheet. Li Jia (1997) established a mixed mode Dugdale-Barenblatt model for a semi-infinite crack in an ideally elastic-plastic thin plate which was loaded by a pair of self-equilibrating concentrated forces, at the crack rims. An analytic solution was obtained for infinite elastic perfectly-plastic plate with center crack loaded by two pairs of point tensile force, by Wang \& Zhou (2004).

Present paper obtains a modified Dugdale model solution for two unequal asymmetric collinear hairline straight cracks weakening an unbounded plate. Remotely applied tensile forces open the faces of the cracks in mode-I deformation developing a plastic zone ahead of each of the tip of the cracks. These developed plastic zones rims are closed by prescribing normal cohesive quadratically varying yield point stress distribution, thus arresting crack from further opening.

\section{Mathematical equations}

According to Muskhelishvili's (1953) complex variable formulation, the stress components $P_{i j}(i, j=x, y)$ may be expressed in terms of two complex potentials $\Phi(z)$ and $\Omega(z)$ as

$$
P_{y y}-i P_{x y}=\Phi(z)+\Omega(\bar{z})-(z-\bar{z}) \Phi^{\prime}(z),
$$

where $\Omega(z)=\Phi(z)+z \Phi^{\prime}(z)+\bar{\Psi}(z)$ and a bar over the function denotes its complex conjugate. Prime after the function denotes its differentiation with respect to the argument of the functions.

Consider an unbounded plate weakened by $\mathrm{n}$ unequal collinear straight cracks $L_{i}(i=$ $1,2,3, \ldots, n)$ with end points $a_{i}, b_{i}$. The configuration so obtained is subjected to following boundary conditions:

(a) $P_{y y}^{ \pm}$act along the rims of the crack $L_{i}(i=1,2,3, \ldots, n)$.

(b) No stresses act at infinite boundary of the plate.

(c) Displacements are single-valued around the rims of cracks.

Boundary condition (a) together with (1) yields following two Hilbert problems

$$
P_{y y}^{+}-i P_{x y}^{+}=\Phi^{+}(t)+\Omega(t)^{-} ; \quad P_{y y}^{-}-i P_{x y}^{-}=\Phi^{-}(t)+\Omega(t)^{+},
$$

under the assumption $\lim _{y \rightarrow 0}\left\{y \Phi^{\prime}(t+i y)\right\}=0$. Superscript + denotes the value of the function when approached from $y>0$ and the superscript - denotes the value of the function when approached from $y<0$.

Solution of (2) may be written, using Muskhelishvili's (1953), as

$$
\Phi(z)=\frac{1}{2 \pi i X(z)} \int_{L} \frac{X(t) p(t)}{t-z} d t+\frac{1}{2 \pi i X(z)} \int_{L} \frac{q(t)}{t-z} d t+\frac{P_{n}(z)}{X(z)},
$$

and

$$
\Omega(z)=\frac{1}{2 \pi i X(z)} \int_{L} \frac{X(t) p(t)}{t-z} d t-\frac{1}{2 \pi i X(z)} \int_{L} \frac{q(t)}{t-z} d t+\frac{P_{n}(z)}{X(z)},
$$


where

$$
\begin{aligned}
X(z) & =\prod_{k=1}^{n}\left(z-a_{k}\right)^{1 / 2}\left(z-b_{k}\right)^{1 / 2}, \quad L=\bigcup_{i=1}^{n} L_{i}, \\
2 p(t) & =\left(P_{y y}^{+}+P_{y y}^{-}\right)-i\left(P_{x y}^{+}+P_{x y}^{-}\right) ; \quad 2 q(t)=\left(P_{y y}^{+}-P_{y y}^{-}\right)-i\left(P_{x y}^{+}-P_{x y}^{-}\right)
\end{aligned}
$$

and

$$
P_{n}(z)=C_{0} z^{n}+C_{1} z^{n-1}+\cdots+C_{n} .
$$

Arbitrary constants $C_{i}(i=1,2,3, \ldots, n)$ are determined using boundary conditions (b) and (c), together with (1 and 5) and single-valuedness condition of displacement, taken from Muskhelishili (1953)

$$
2(\kappa+1) \int_{L} \frac{P_{n}(t)}{X(t)} d t+\kappa \int_{L}\left[\Phi_{0}^{+}(t)-\Phi_{0}^{-}(t)\right] d t+\int_{L}\left[\Omega_{0}^{+}(t)-\Omega_{0}^{-}(t)\right] d t=0 .
$$

\section{Statement of the problem}

An infinite homogeneous elastic perfectly-plastic plate assumed to occupy xoy-plane. The plate is weakened by two unequal collinear hairline straight cuts $L_{1}$ and $L_{2}$ occupy the intervals $\left[d_{1}, c_{1}\right]$ and $\left[b_{1}, a_{1}\right]$, on $o x$-axis, respectively. A uniform constant tension, $\sigma_{\infty}$, is applied at infinite boundary in a direction perpendicular to the rims of the cracks. Consequently, the faces of the cracks open in Mode-I type deformations forming a plastic zones $\Gamma_{1}, \Gamma_{2}, \Gamma_{3}$ and $\Gamma_{4}$ ahead of each tip $d_{1}, c_{1}, b_{1}$ and $a_{1}$ of the cracks, respectively. Each of the plastic zone $\Gamma_{1}, \Gamma_{2}, \Gamma_{3}$ and $\Gamma_{4}$ occupy the interval $\left[d, d_{1}\right],\left[c_{1}, c\right],\left[b, b_{1}\right]$ and $\left[a_{1}, a\right]$, respectively, along the $o x$-axis. Rims of these plastic zones are subjected to the stress $P_{y y}^{ \pm}=t^{2} \sigma_{y e}, P_{x y}^{ \pm}=0$, where $\sigma_{y e}$ denotes yield point stress of the plate and $\mathrm{t}$ is any point on any of the plastic zone, $\Gamma_{i}(i=1,2,3,4)$. Figure 1 shows the configuration of the problem.

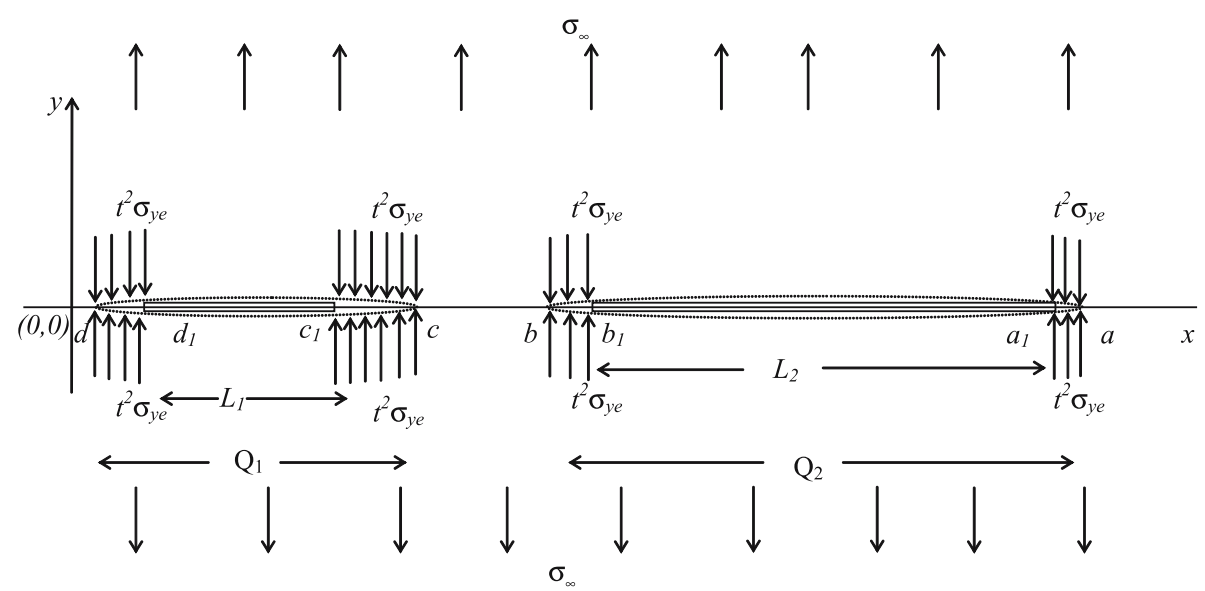

Figure 1. Schematic representation of the configuration of the problem. 


\section{Solution of the problem}

The above problem is solved using principle of superposition of the stress intensity factors for two component problems contributing towards the stress singularity. These two problems are appropriately derived from the above stated problem and are termed as Problem-I and Problem-II.

\subsection{Statement and solution of problem-I}

An infinite homogeneous elastic perfectly-plastic plate is weakened by two unequal hairline collinear straight cracks $Q_{1}$ and $Q_{2}$. The crack: $Q_{1}\left(=\Gamma_{1} U L_{1} U \Gamma_{2}\right)$ occupies the interval $[d, c]$ and crack $Q_{2}\left(=\Gamma_{3} U L_{2} U \Gamma_{4}\right)$ occupies the interval $[b, a]$ along $o x$-axis. Boundary conditions of the problems are

(i) Rims of the cracks $Q_{1}$ and $Q_{2}$ are stress free.

(ii) Infinite boundary of the plate is subjected to stress distribution $P_{y y}^{ \pm}=\sigma_{\infty}, P_{x y}^{ \pm}=0$.

(iii) Displacements are single-valued around the rims of cracks $Q_{1}$ and $Q_{2}$.

Using boundary conditions (i), (iii) and (3), the desired potential $\Phi^{I}(z)$ may be written as

$$
\Phi^{I}(z)=\frac{C_{0} z^{2}+z C_{1}+C_{2}}{X(z)}-\frac{\sigma_{\infty}}{4},
$$

where superscript $I$ denotes that the function refers to Problem-I

$$
\begin{aligned}
& C_{0}= \frac{\sigma_{\infty}}{2}, \quad C_{1}=-\frac{\sigma_{\infty}}{2} \frac{\left|\begin{array}{cc}
Y_{2} & Y_{0} \\
W_{2} & W_{0}
\end{array}\right|}{\left|\begin{array}{ll}
Y_{1} & Y_{0} \\
W_{1} & W_{0}
\end{array}\right|}=\frac{\sigma_{\infty}}{2} K_{1}, \quad C_{2}=-\frac{\sigma_{\infty}}{2} \frac{\left|\begin{array}{cc}
Y_{1} & Y_{2} \\
W_{1} & W_{2}
\end{array}\right|}{\left|\begin{array}{cc}
Y_{1} & Y_{0} \\
W_{1} & W_{0}
\end{array}\right|}=\frac{\sigma_{\infty}}{2} K_{2} \\
& X(z)=\sqrt{z-a} \sqrt{z-b} \sqrt{z-c} \sqrt{z-d} \\
& W_{0}=g V_{0}, \quad W_{1}=g\left[a V_{0}+(d-a) V_{1}\right], \quad W_{2}=g\left[a^{2} V_{0}+2 a(d-a) V_{1}+(d-a)^{2} V_{2}\right] \\
& V_{0}= F(k), \quad V_{1}=\Pi\left(\alpha^{2}, k\right), \\
& V_{2}= \frac{1}{2\left(\alpha^{2}-1\right)\left(k^{2}-\alpha^{2}\right)}\left[\alpha^{2} E(k)+\left(k^{2}-\alpha^{2}\right) F(k)\right. \\
&\left.+\left(2 k^{2} \alpha^{2}+2 \alpha^{2}-\alpha^{4}-3 k^{2}\right) \Pi\left(\alpha^{2}, k\right)\right] \\
& g= \frac{2}{\sqrt{a-c} \sqrt{b-d}}, \quad \alpha^{2}=\frac{d-c}{a-c}<0, \quad k^{2}=\frac{(a-b)(c-d)}{(a-c)(b-d)} \\
& Y_{2}= g\left[c^{2} U_{0}+2 c(b-c) U_{1}+(b-c)^{2} U_{2}\right], \quad Y_{1}=g\left[c U_{0}+(b-c) U_{1}\right], \quad Y_{0}=g U_{0}, \\
& \beta^{2}= \frac{a-b}{a-c}<1, \quad \varphi=\frac{\pi}{2}, \quad U_{0}=F(k), \quad U_{1}=\Pi\left(\beta^{2}, k\right), \\
& U_{2}= \frac{1}{2\left(\beta^{2}-1\right)\left(k^{2}-\beta^{2}\right)}\left[\beta^{2} E(k)+\left(k^{2}-\beta^{2}\right) F(k)\right. \\
&\left.+\left(2 k^{2} \beta^{2}+2 \beta^{2}-\beta^{4}-3 k^{2}\right) \Pi\left(\beta^{2}, k\right)\right]
\end{aligned}
$$

and $F(k), \Pi\left(\alpha^{2}, k\right)$ are the complete elliptic integral of first and third kind, respectively. 


\subsection{Statement and solution of problem-II}

Configuration of the problem remains the same as stated in section $4 \cdot 1$. The boundary conditions for this problem are as follows:

(p) Segments $\Gamma_{i}(i=1,2,3,4)$ of cracks $Q_{1}$ and $Q_{2}$ are subjected to stress distribution $P_{y y}^{ \pm}=t^{2} \sigma_{y e}, P_{x y}^{ \pm}=0$.

(q) Segments $L_{i}(i=1,2)$ of cracks $Q_{1}$ and $Q_{2}$ are stress free.

(r) Infinite Boundary of the plate is stress free.

(s) Displacements are single-valued around cracks $Q_{1}$ and $Q_{2}$.

Boundary conditions (p) and (q) when substituted in (1) yield following two Hilbert problems

$$
\begin{aligned}
& \left\{\Phi^{I I}(t)+\Omega^{I I}(t)\right\}^{+}+\left\{\Phi^{I I}(t)+\Omega^{I I}(t)\right\}^{-}=2 t^{2} \sigma_{y e}, \\
& \left\{\Phi^{I I}(t)-\Omega^{I I}(t)\right\}^{+}-\left\{\Phi^{I I}(t)-\Omega^{I I}(t)\right\}^{-}=0,
\end{aligned}
$$

where superscript $I I$ denotes that the function refers to Problem II. Desired potential $\Phi^{I I}(z)$ may be written using (3) putting $p(t)=t^{2} \sigma_{y e}$ and $q(t)=0$ and integrating

$$
\Phi^{I I}(z)=\frac{\sigma_{y e}}{2 \pi i X(z)} \int_{\bigcup_{i=1}^{4} \Gamma_{i}} \frac{t^{2} X(t)}{t-z} d t+\frac{D_{1} z+D_{2}}{X(z)},
$$

where $X(z)$ is same as given by (7). Integral is evaluated using Byrd \& Friedman (1954). $D_{1}$ and $D_{2}$ are obtained using boundary conditions (r) and (s) together with $(1,5,9)$.

The function $\Phi^{I I}(z)$

$$
\Phi^{I I}(z)=\frac{\sigma_{y e}}{2 \pi X(z)}\left\{M+z N+z^{2} F+z^{3} G+X^{2}(z) H+z X^{2}(z) J_{0}\right\}+f(z)
$$

where

$$
\begin{aligned}
& M=-Q-H_{4} E-H_{1} H_{4} J_{0}, \quad N=-P+H_{3} E-H_{4} J_{0}+H_{1} H_{3} J_{0}, \\
& F=C-H_{2} E+H_{3} J_{0}-H_{1} H_{2} J_{0}, \quad G=D+H_{1} E-H_{2} J_{0}+H_{1}^{2} J_{0}, \\
& H=E+H_{1} J_{0} \quad A=\left\{J_{5}-H_{1} J_{4}+H_{2} J_{3}-H_{3} J_{2}+H_{4} J_{1}\right\} \\
& B=\left\{J_{4}-H_{1} J_{3}+H_{2} J_{2}-H_{3} J_{1}+H_{4} J_{0}\right\} \\
& C=J_{3}-H_{1} J_{2}+H_{2} J_{1}-H_{3} J_{0}, \quad D=J_{2}-H_{1} J_{1}+H_{2} J_{0}, \quad E=J_{1}-H_{1} J_{0}, \\
& H_{1}=a+b+c+d, \quad H_{2}=a b+a c+a d+b c+b d+c d, \\
& H_{3}=a b c+a b d+a c d+b c d, \quad H_{4}=a b c d \\
& J_{k}=\left(R_{k}+S_{k}-T_{k}-U_{k}\right), \quad k=0,1,2,3,4,5 \\
& R_{k}=\int_{d}^{d_{1}} \frac{t^{k}}{\sqrt{a-t} \sqrt{b-t} \sqrt{c-t} \sqrt{t-d}} d t, \quad S_{k}=\int_{c_{1}}^{c} \frac{t^{k}}{\sqrt{a-t} \sqrt{b-t} \sqrt{c-t} \sqrt{t-d}} d t \\
& T_{k}=\int_{b}^{b_{1}} \frac{t^{k}}{\sqrt{a-t} \sqrt{t-b} \sqrt{t-c} \sqrt{t-d}} d t, \quad U_{k}=\int_{a_{1}}^{a} \frac{t^{k}}{\sqrt{a-t} \sqrt{t-b} \sqrt{t-c} \sqrt{t-d}} d t
\end{aligned}
$$




$$
\begin{aligned}
& P=C \frac{\left[\frac{W_{2}}{W_{0}}-\frac{Y_{2}}{Y_{0}}\right]}{\left[\frac{W_{1}}{W_{0}}-\frac{Y_{1}}{Y_{0}}\right]}+D \frac{\left[\frac{W_{3}}{W_{0}}-\frac{Y_{3}}{Y_{0}}\right]}{\left[\frac{W_{1}}{W_{0}}-\frac{Y_{1}}{Y_{0}}\right]}+E \frac{\left[\frac{W_{4}}{W_{0}}-\frac{Y_{4}}{Y_{0}}\right]}{\left[\frac{W_{1}}{W_{0}}-\frac{Y_{1}}{Y_{0}}\right]}+J_{0} \frac{\left[\frac{W_{5}}{W_{0}}-\frac{Y_{5}}{Y_{0}}\right]}{\left[\frac{W_{1}}{W_{0}}-\frac{Y_{1}}{Y_{0}}\right]} \\
& Q=C \frac{\left[\frac{W_{2}}{W_{1}}-\frac{Y_{2}}{Y_{1}}\right]}{\left[\frac{W_{0}}{W_{1}}-\frac{Y_{0}}{Y_{1}}\right]}+D \frac{\left[\frac{W_{3}}{W_{1}}-\frac{Y_{3}}{Y_{1}}\right]}{\left[\frac{W_{0}}{W_{1}}-\frac{Y_{0}}{Y_{1}}\right]}+E \frac{\left[\frac{W_{4}}{W_{1}}-\frac{Y_{4}}{Y_{1}}\right]}{\left[\frac{W_{0}}{W_{1}}-\frac{Y_{0}}{Y_{1}}\right]}+J_{0} \frac{\left[\frac{W_{5}}{W_{1}}-\frac{Y_{5}}{Y_{1}}\right]}{\left[\frac{W_{0}}{W_{1}}-\frac{Y_{0}}{Y_{1}}\right]} \\
& f(z)=\frac{X(z) \sigma_{y e}}{2 \pi i} \int_{i=1}^{4} \Gamma_{i}^{\frac{z^{2}}{(t-z) X(t)} d t} .
\end{aligned}
$$

\section{Plastic zone length}

Lengths of each plastic zone are obtained using the Dugdale hypothesis that stresses remain finite at every point of the body yields

$$
\frac{\sigma_{\infty}}{\sigma_{y e}}=-\frac{\left(M+z N+z^{2} F+z^{3} G\right)}{\pi\left(z^{2}+z K_{1}+K_{2}\right)} .
$$

Four non-linear equations are obtained from the above conditions which enables to determine $a, b, c$ and $d$ for prescribed values of $a_{1}, b_{1}, c_{1}, d_{1}$, and $\frac{\sigma_{\infty}}{t^{2} \sigma_{v e}}$. Plastic zones are then calculated using the relation $\left|d_{1}-d\right|,\left|c-c_{1}\right|,\left|b_{1}-b\right|$ and $\left|a-a_{1}\right|$.

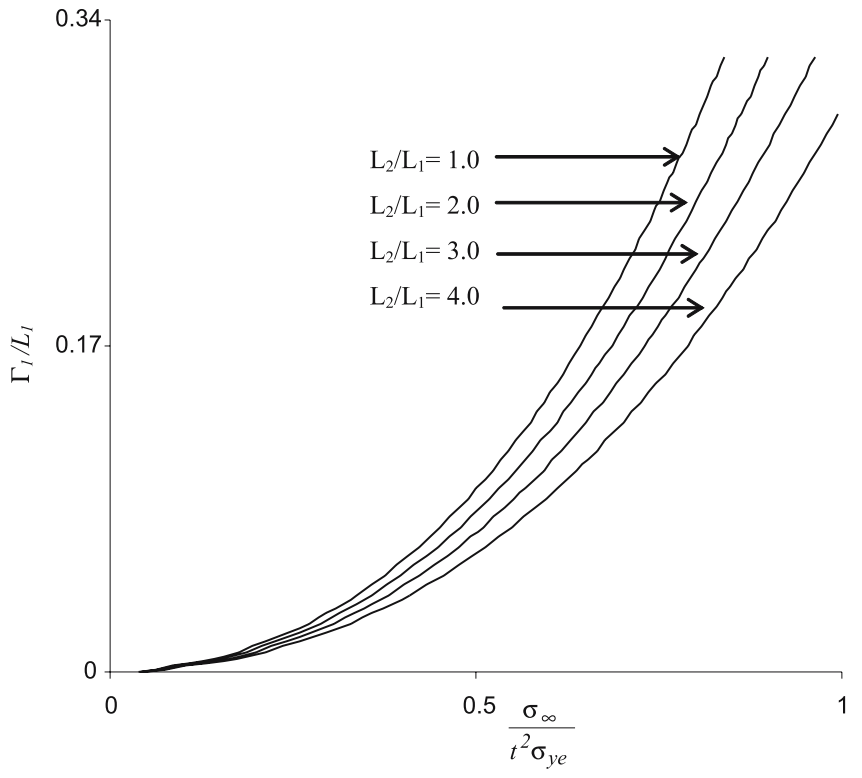

Figure 2. Variation between load ratio and a ratio of plastic zone length at the outer tip $d_{1}$ of the smaller crack. 


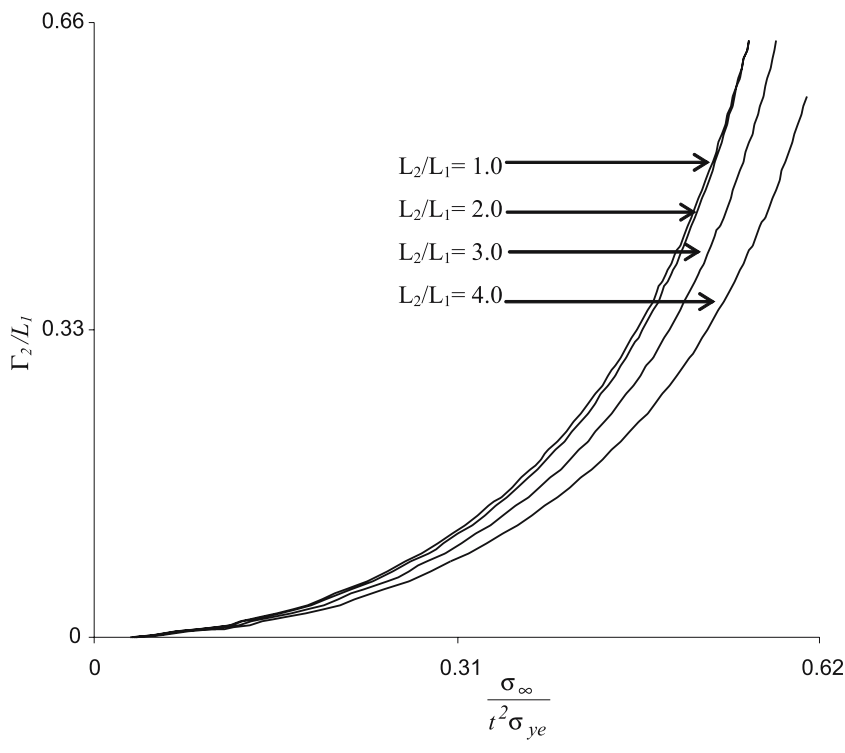

Figure 3. Variation between load ratio and a ratio of plastic zone length at the inner tip $c_{1}$ of the smaller crack.

\section{Case study}

An illustrative example is presented to study the variation of applied load ratio $\frac{\sigma_{\infty}}{t^{2} \sigma_{\text {ve }}}$ (load applied at infinite boundary to load applied on the developed plastic zone) versus ratio of developed plastic zone to relevant crack length $\frac{\Gamma_{i}}{L_{i}}$. The investigations are carried out for different crack length ratios, $\frac{L_{2}}{L_{1}}$, starting from the case of two cracks being of equal in length to the case when the bigger crack length being four times that of the smaller crack length. For the case when crack lengths are equal, the results match with the results of Theocaris (1983).

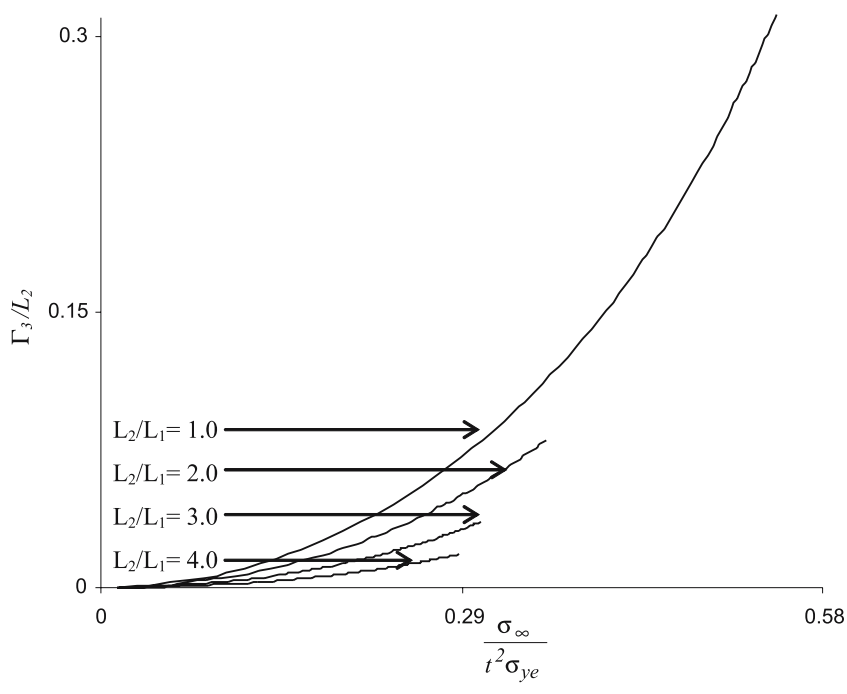

Figure 4. Variation between load ratio and a ratio of plastic zone length at the inner tip $b_{1}$ of the bigger crack. 


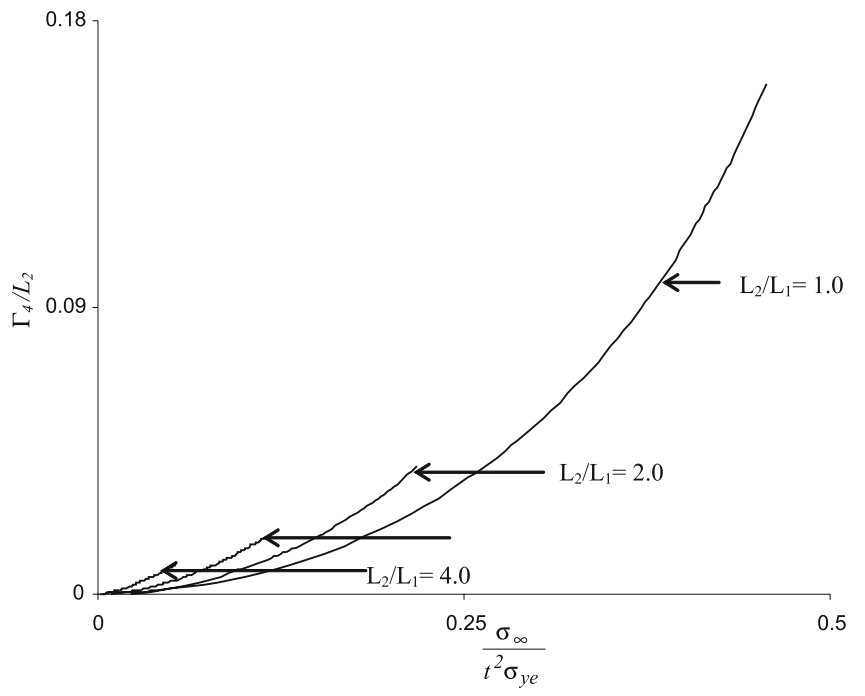

Figure 5. Variation between load ratio and a ratio of plastic zone length the outer tip $a_{1}$ of the bigger crack.

Figure 2 depicts the variations of $\frac{\Gamma_{1}}{L_{1}}$ with respect to load ratio $\frac{\sigma_{\infty}}{t^{2} \sigma_{y}}$, when it is varied from zero to one at the tip $d_{1}$ of smaller crack $L_{1}$. A parabolic increased is observed in load required to arrest crack opening as the plastic zone is increased. If the lengths of cracks are made unequal then comparatively less load is required to crack arrest although the behaviour remains the same.

Now, for this variation of plastic zone at the crack tip $d_{1}$ (is the maximum load sustainability at tip $d_{1}$ ) the variation plastic zone length at the three remaining tips of the cracks is investigated

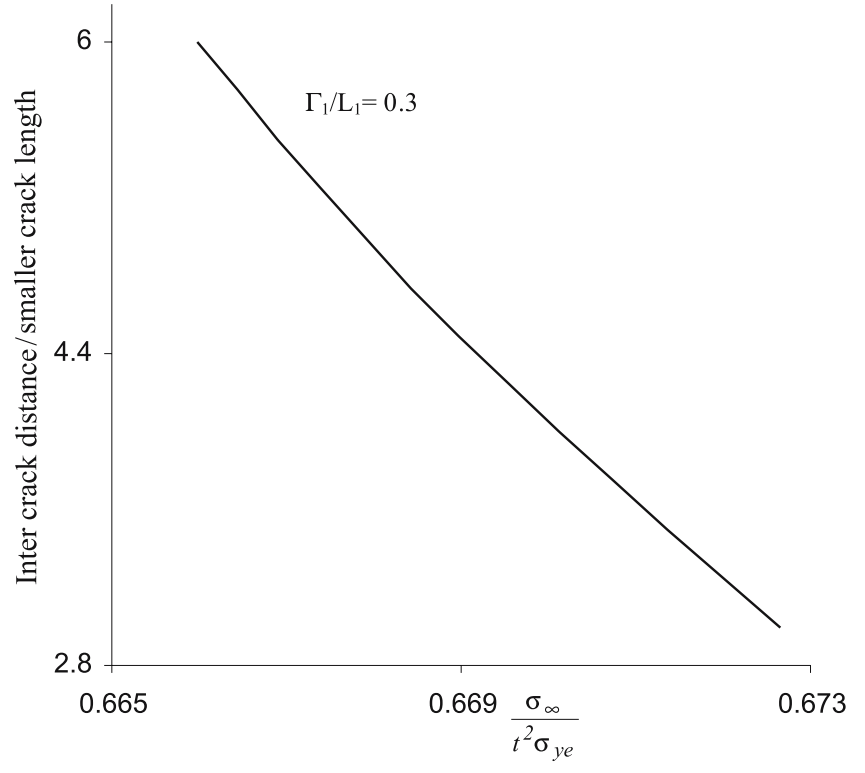

Figure 6. Variation of load required with respect to inter crack distance at the exterior tip $d_{1}$ of the smaller. 


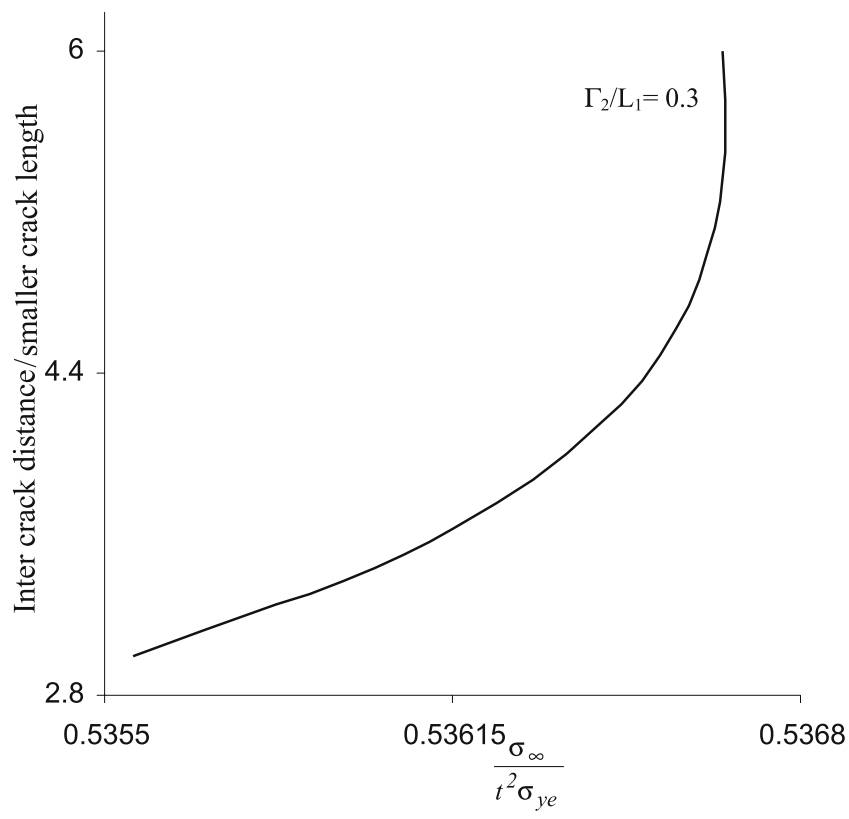

Figure 7. Variation of load required with respect to inter crack distance at the interior tip $c_{1}$ of the smaller crack.

with respect to $\frac{\sigma_{\infty}}{t^{2} \sigma_{v e}}$. The inner tip $c_{1}$ of the smaller crack is most distressed situation as shown in figure 3 . The load bearing capacity at this tip is almost sixty percent less than at the tip $d_{1}$ and the plastic zone developed at tip $d_{1}$ is almost half the size of the plastic zone developed at the tip $c_{1}$. As plastic zone size is increased the load required to arrest the plastic zones increased exponentially.

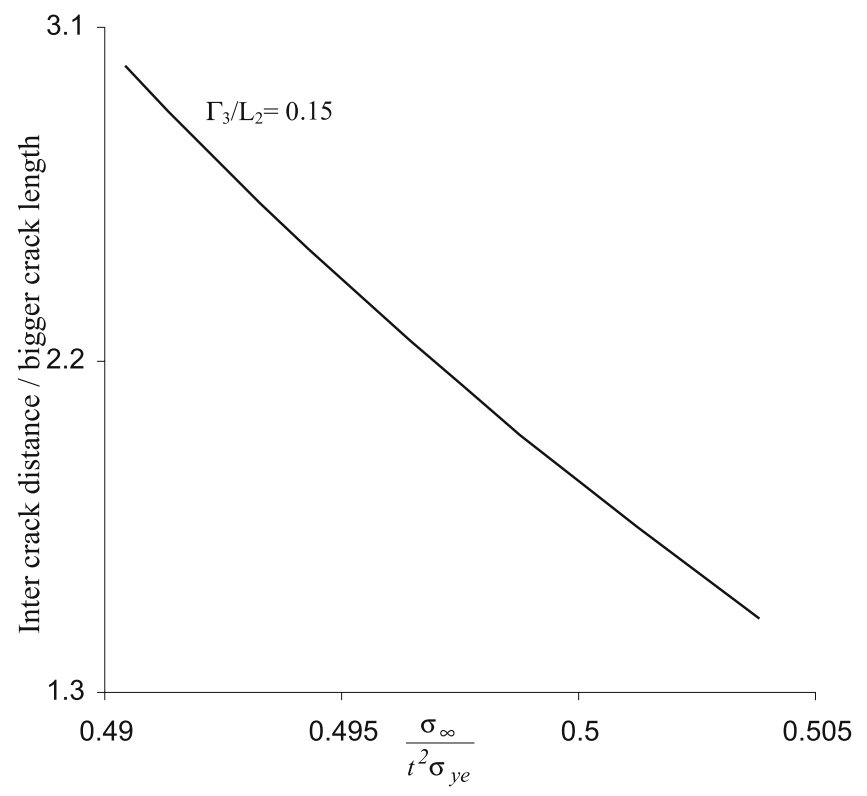

Figure 8. Variation of load required with respect to inter crack distance at the inner tip $b_{1}$ of the bigger crack. 


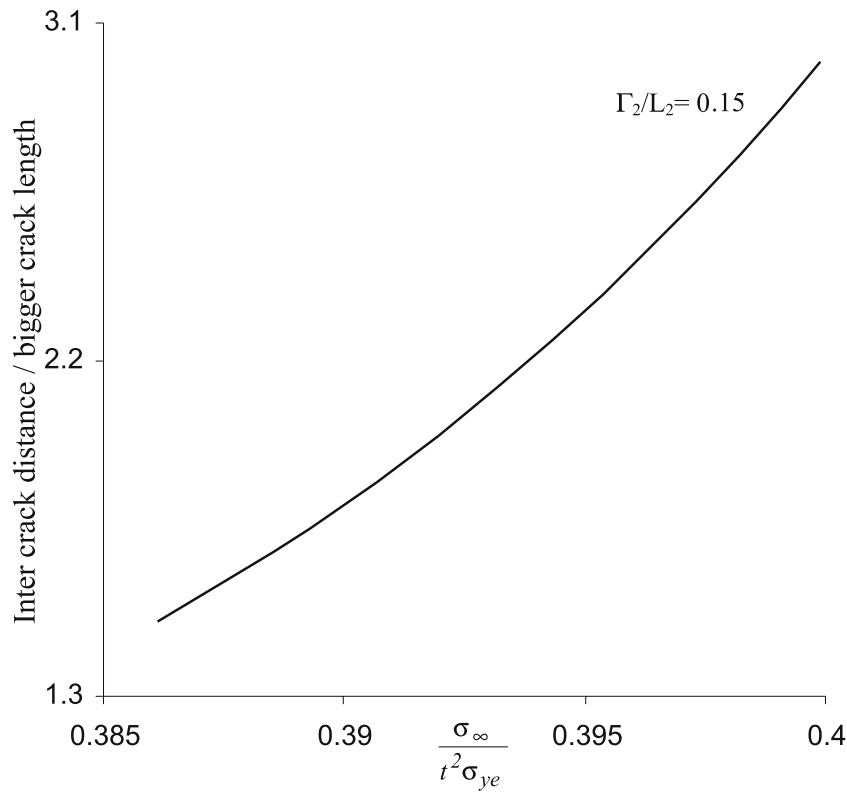

Figure 9. Variation of load required with respect to inter crack distance at the interior tip $c_{1}$ of the smaller crack.

Figure 4 shows the variation of $\frac{\sigma_{\infty}}{t^{2} \sigma_{y e}}$ against $\frac{\Gamma_{3}}{L_{1}}$ (plastic zone increases at the inner tip $b_{1}$ of the bigger crack). For the maximum load bearing capacity at the tip $d_{1}$, it is observed that almost half the load is required to arrest the crack at this inner tip of the bigger crack as compared to that at the exterior tip $d_{1}$ of the smaller crack. As crack length ratio $\frac{L_{2}}{L_{1}}$ is increased, the developed crack zone size is much reduced and load required to arrest the developed plastic zone also reduced drastically.

While figure 5 shows that the smallest plastic zone develops at this exterior tip of the bigger crack tip. This far end tip remains most unaffected. As the length of the bigger crack is increased a very small plastic zone develops at this tip, as expected.

Figures 6, 7, 8 and 9 show the variation of load required to arrest the crack opening as the inter crack distance between the two cracks is increased. It may be noted that the behaviour of load ratio to inter-crack distance/crack length at the tip $d_{1}, b_{1}$ is similar, although the plastic zone is bigger at the tip of smaller crack. It is important to point out that the behaviour of the load required to arrest the crack at the interior tips of the bigger and smaller cracks is opposite to each other, as expected.

Comparing the behaviour at the interior tips of the bigger and smaller crack it is observed that the interior tip of the smaller crack is most stressed and shows a parabolic behaviour while the interior tip of the bigger crack shows a closing behaviour but the variation is comparatively much linear.

The authors are grateful to Prof. R D Bhargava [Senior Professor and Head (retd.), Dept. of Mathematics, Indian Institute of Technology, Bombay] for his valuable suggestions during the course of this work. The authors are also grateful to the referees for their suggestions to improve the understandability of the paper. 


\section{References}

Beretta S, Carboni M 2005 A strip-yield algorithm for the analysis of closure evaluation near the crack tip. Eng. Fracture Mech. 72: 1222-1237

Bhargava R R, Hasan S 2001 Arrest of opening of two circular arc cracks with coalesced plastic zones. J. Mech. Composite Materials 37: 201-206

Byrd P F, Friedman M D 1954 Hand Book of Elliptic Integrals for Engineers and physicists (Lange, London: Maxwell \& Springer Ltd.)

Collins R A, Cartwright D J 2001 An analytical solution for two equal-length collinear strip yield cracks. Eng. Fracture Mech. 68: 915-924

Dugdale D S 1960 Yielding of sheets containing slits. J. Mechanics and Physics of Solids 8: 100-104

Harrop L P 1978 Application of a modified Dugdale model to the K vs. COD relation. Eng. Fracture Mech. 10: 807-816

Li Jia 1997 A Dugdale-Barenblatt model for a plane stress semi-infinite crack under mixed mode concentrated forces. Int. J. Fracture 88: 153-166

Muskhelishvili N I 1953 Some basic problems of the mathematical theory of elasticity. Groningen P. Noordhoff Ltd. (English translation) Netherlands

Nishimura Toshihiko 2002 Strip yield analysis of two collinear unequal cracks in an infinite sheet. Eng. Fracture Mechanics 69 1173-1191

Theocaris P S 1983 Dugdale model for two collinear unequal cracks. Eng. Fracture Mech. 18: 545-559

Wang C H, Rose L R F, Chen G X 2002 A strain-based Dugdale model for cracks under generally yielding conditions. Int. J. Fracture 113: 77-104

Wang Jian-hua, Zhou Xiao-ping 2004 Near crack line elastic-plastic analysis for a infinite plate loaded by two pairs of point tensile forces. Mech. Res. Commun. 31: 415-420 